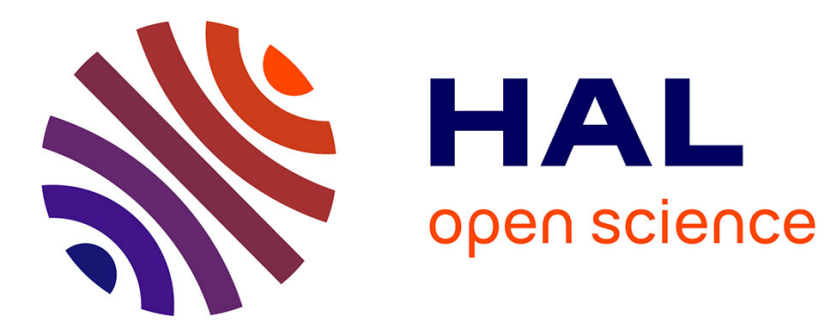

\title{
Interactions of blood proteins with poly(isobutylcyanoacrylate) nanoparticles decorated with a polysaccharidic brush
}

Denis Labarre, Christine Vauthier, Cédric Chauvierre, Boris Petri, R Muller, Mohamed M Chehimi

\section{To cite this version:}

Denis Labarre, Christine Vauthier, Cédric Chauvierre, Boris Petri, R Muller, et al.. Interactions of blood proteins with poly(isobutylcyanoacrylate) nanoparticles decorated with a polysaccharidic brush. Biomaterials, 2005, 26 (24), pp.5075 - 5084. 10.1016/j.biomaterials.2005.01.019 . hal-03206191

\section{HAL Id: hal-03206191 \\ https://hal.science/hal-03206191}

Submitted on 23 Apr 2021

HAL is a multi-disciplinary open access archive for the deposit and dissemination of scientific research documents, whether they are published or not. The documents may come from teaching and research institutions in France or abroad, or from public or private research centers.
L'archive ouverte pluridisciplinaire HAL, est destinée au dépôt et à la diffusion de documents scientifiques de niveau recherche, publiés ou non, émanant des établissements d'enseignement et de recherche français ou étrangers, des laboratoires publics ou privés. 


\title{
Interactions of blood proteins with poly (isobutylcyanoacrylate) nanoparticles decorated with a polysaccharidic brush
}

\author{
Denis Labarre $^{a *}$, Christine Vauthier ${ }^{a}$, Cédric Chauvierre ${ }^{a}$, Boris Petri ${ }^{b}$, Rainer \\ H. Müller ${ }^{b}$ and Mohamed M. Chehimic
}

a Laboratoire de Physico-Chimie, Pharmacotechnie et Biopharmacie, UMR CNRS 8612, Université Paris Sud-XI, 92296 Châtenay-Malabry Cedex, France. ' Department of Pharmacy, Freie Universität Berlin, 12169 Berlin, Germany. 'Interfaces, Traitements, Organisation et Dynamique des Systèmes (ITODYS), UMR CNRS 7086, Université Paris 7, 1 rue Guy de la Brosse,75005 Paris, France

Published in: Biomaterials. 2005;26(24):5075-84. https://doi.org/10.1016/j.biomaterials.2005.01.019

\section{*Correspondence.}

Laboratoire de Physico-Chimie, Pharmacotechnie et Biopharmacie, UMR CNRS 8612, Centre d'études pharmaceutiques, Université Paris Sud-XI, 5 rue J.B. Clément, 92296 Châtenay-Malabry Cedex, France. E-mail: Denis.labarre@cep.u-psud.fr, Tel: +33 146835 708, Fax: +33 146835312

\begin{abstract}
:
The aim of this work was to examine the in vitro interactions of core-shell poly (isobutylcyanoacrylate)-polysaccharide nanoparticles (NP) with blood proteins. The particles were prepared by initiating the emulsion polymerisation of isobutylcyanoacrylate (IBCA) in the presence of dextran 71 or $15 \mathrm{kDa}$, heparin, a blend of dextran 71 and heparin, or dextran sulphate in aqueous medium at $\mathrm{pH}$ 1. The mechanisms of polymerisation were redox radical (Rad) or anionic (An), resulting in differences in the spatial arrangement of the polysaccharide chains at the NP surface, i.e. "loops" and "trains" by anionic polymerization, "brush" by radical polymerization. Surface composition of NPs was determined by X-ray photo-electron spectroscopy (XPS) and surface charge by zeta potential measurements. In the presence of citrated blood plasma, efficacy of the steric repulsive effect of the NP dextran shell towards protein adsorption decreased in the order: Dex71Rad > Dex15-Rad > Dex71-An >> Dex15-An. Dextran-coated NPs adsorbed ApoA-I and fibrinogen from plasma. Concerning activation of complement in serum, the effect was sharp: Dex71-Rad was a very low activator whereas Dex15-An, Dex15-Rad and Dex71-An were strong activators. In citrated plasma, the steric repulsive effects of Hep-Rad and Dex-Hep-Rad NPs were similar to Dex71-An, and Dex-Sulph-Rad NPs adsorbed twice more proteins than Hep-Rad. Hep-Rad, Dex-Hep-Rad and DexSulph-Rad NPs adsorbed IgG and fibrinogen. Complement was not activated in serum in the presence of Hep-Rad and Dex-Hep-Rad and a slight adsorption of C3 was noted. C3 was completely adsorbed on Dex-Sulph-Rad. The exquisite sensitivity of blood proteins to differences in the nature and outermost structure of the polysaccharides-coated NPs is highlighted by the present results.
\end{abstract}

Keywords: Core-shell nanoparticles; poly (isobutylcyanoacrylate); dextran; heparin; dextran sulphate; surface properties; XPS; blood plasma proteins adsorption; complement activation 


\section{INTRODUCTION}

After intravascular administration, drugs are distributed throughout the body as a function of their physico-chemical properties, resulting in contamination of non-targeted tissues. Controlling the biodistribution of a drug is thus one of the main challenges for the design of polymeric nanoparticulate drug carrier systems.

In vivo, the fate of nanoparticles (NPs) used as drug carriers results from their surface properties. As any foreign body, intravenously injected "conventional" NPs are opsonised and taken up from the blood circulation by phagocytes, including those of the mononuclear phagocytic system. This effect hinders possibilities of specific delivery of drugs to other tissues. Thus, NPs made of amphiphilic block copolymers able to self-organise into core-shell particles in aqueous solvents were designed to reduce opsonisation and phagocytosis. The most popular compound used to make the hydrophilic part of these copolymers was poly (ethylene oxide) (PEO) which either constituted the shell of NPs or was used to create protein repulsive surfaces in contact with blood [1]. Therefore, long blood circulating NPs could be obtained $[\mathbf{2}, \mathbf{3}]$. This property resulted from steric repulsion of proteins induced by the hydrophilic chains of the NP shell [4-6]. In the presence of NPs composed of a core of polylactides (PLA) bearing a "brush" of PEO (PLA-PEO), the rate of complement activation was reduced, and plasma factors of coagulation were not activated when compared to "conventional" PLA NPs stabilised by other surfactants $[7,8]$. PEO can be replaced by other hydrophilic polymers. Indeed, protein adsorption was reduced on NPs coated with polyacrylamide, poly(vinylpyrrolidone) or dextran brushes suggesting that highly water soluble and flexible polymer chains grafted on the NPs surface can be efficient to reduce opsonisation [9-11]. The chemical composition of the macromolecule grafted on the NPs surface seemed to be not the only factor affecting the fate of the carrier after intravenous administration. Some effects related to the morphology of the outermost structure, either "in brush", or "in loops", were also reported $[12,13]$. The complement activations produced by NPs made of either diblock or triblock copolymers of poly (alkyl cyanoacrylate) (PACA) and PEO were markedly different [13].

Polysaccharides are recognised for having a high potential in the development of long-circulating and potentially even of targeting drug delivery systems [10-12,14-15]. The properties of NPs with a polysaccharide shell depend on the type of the bound polysaccharide. For instance, it was shown that core-shell NPs made of a poly (methyl methacrylate) (PMMA) core and of a heparin shell did not activate complement and could circulate for hours when injected to mice, in opposition to "conventional" PMMA NPs [16]. However, the success of the approach can also strongly depend on structural parameters of the outermost surface, as exemplified by the case of dextran. Soluble dextran has been used for years in humans as blood plasma substitute. Conversely, crosslinked dextran microparticles such as Sephadex are powerful activators of the complement system [17]. However, a complement activation level as low as the one observed with soluble dextran was reported with core-shell NPs made of a PMMA core on which dextran chains were bound by only one end [18]. These NPs were long circulating [16] emphasizing the importance of both the composition and the structural arrangement of the macromolecular chains in the shell of the NPs for biological recognition phenomena of foreign bodies. The effect of the structural arrangement of the chains at the NP surface was further evidenced in a very recent work considering polyester NPs coated with polysaccharides [19]. In this work, the polysaccharide chains at the NP surface adopted a configuration in loops due to the grafting of a few polyester lipophilic anchors on one polysaccharide chain [20]. These NPs induced a strong activation of the complement [19] conversely to the NPs with polysaccharide chains attached by one end [18].

The purpose of the work presented in the present paper is to investigate how the presence in the shell of NPs of polysaccharides differing in structural and chemical characteristics, may affect the properties of the NPs surface towards blood proteins. To this aim, biodegradable NPs were prepared by emulsion polymerization of isobutylcyanoacrylate using different polysaccharides as initiators of a 
redox radical polymerization $[\mathbf{2 1}, \mathbf{2 2}]$ or of an anionic polymerisation [23]. The surface chemistry of dried NPs was studied by means of X-ray photo-electron spectroscopy (XPS). The surface charge of NPs in aqueous suspension was investigated by means of zeta potential measurements. Complement activation induced by incubation of the NPs with human serum was evaluated by conversion of C3 as evidenced by two-dimensional immunoelectrophoresis. Adsorption of blood proteins onto the NP surface after incubation in citrated plasma was evaluated by two-dimensional polyacrylamide gel electrophoresis (2-D PAGE) of the desorbed proteins and fragments.

\section{MATERIALS AND METHODS}

\subsection{Materials}

Isobutylcyanoacrylate (IBCA) was kindly provided by Loctite (Dublin, Ireland). The polysaccharides were dextran 15 kDa (Dex15), dextran 71 kDa (Dex71), heparin 19 kDa (Hep), obtained from Sigma (Saint-Quentin Fallavier, France), and dextran sulphate $40 \mathrm{kDa}$ (Dex-Sulph), obtained from ICN Pharmaceuticals (Costa Mesa, CA, USA). Other chemicals were reagent grade and used as obtained.

\subsection{Preparation of the nanoparticles:}

A polysaccharide $(0.1375 \mathrm{~g})$, or a blend of Dex71 (0.0688g) and Hep $(0.0688 \mathrm{~g})$, was dissolved in $8 \mathrm{~mL}$ of aqueous $0.2 \mathrm{M} \mathrm{HNO}_{3}$ and the solution was placed under slow speed magnetic stirring and argon bubbling at $40^{\circ} \mathrm{C}$. After $10 \mathrm{~min}, 2 \mathrm{~mL}$ of a solution of cerium (IV) ammonium nitrate $\left(8.10^{-2} \mathrm{M}\right.$ in aqueous $0.2 \mathrm{M} \mathrm{HNO}_{3}$ ) and $0.5 \mathrm{~mL}$ of IBCA were successively added. Argon bubbling was maintained for $10 \mathrm{~min}$. The reaction was left to continue for $40 \mathrm{~min}$ at $40^{\circ} \mathrm{C}$. After cooling to room temperature, $1.25 \mathrm{~mL}$ of an aqueous solution of $1 \mathrm{M}$ trisodium citrate was added and the $\mathrm{pH}$ was adjusted to 7.4 with aqueous $1 \mathrm{M} \mathrm{NaOH}$. The NPs suspension was purified three times by dialysis against distilled water (1L), using Spectra/Por membranes with a cut-off of $100 \mathrm{kDa}$ (Biovalley, Marne la Vallée, France), and stored at $4^{\circ} \mathrm{C}$ until use. NPs suspensions resulting from the redox radical polymerization initiated by both the polysaccharide and cerium ions were named Dex15-Rad, Dex71-Rad, Hep-Rad, Dex71-Hep-Rad and Dex-Sulph-Rad depending on the type of polysaccharide used for the synthesis.

The anionic polymerisation was initiated in acidified aqueous solutions of dextran. Briefly, dextran (0.1375g), either Dex15, or Dex 71 , was dissolved in $10 \mathrm{~mL}$ of aqueous $0.2 \mathrm{M} \mathrm{HNO}_{3}$, and the solution was placed under slow speed magnetic stirring and argon bubbling at $40^{\circ} \mathrm{C}$. After $10 \mathrm{~min}, 0.5 \mathrm{~mL}$ of IBCA were added and the polymerization was continued according to the same protocol as described above. Suspensions of particles obtained by the anionic polymerization were named Dex71-An and Dex15-An.

\subsection{Determination of the concentration of nanoparticles in the suspensions and of the elemental composition of the nanoparticles}

The concentration of NPs was evaluated by gravimetric determination: $1 \mathrm{~g}$ of the purified NPs suspensions was freeze-dried and the dry residue composed of the NPs was weighted. Composition of the NPs was determined by elemental analysis of carbon, hydrogen, oxygen, nitrogen, sulphur and sodium in the dry residue (Service Central d'Analyses du CNRS, Vernaison, France)

\subsection{XPS measurements}

A Surface Science Instrument (SSI) spectrometer (based at ITODYS Laboratory, University Paris VII) equipped with a monochromatic Al K $\alpha$ X-ray source $(1486.6 \mathrm{eV})$ was used at a spot size of $1000 \mu \mathrm{m}$. The take-off angle relative to the sample holder surface was $35^{\circ}$. The pressure in the analysis chamber was ca. $5 \cdot 10^{-9}$ mbar. The pass energy was set at 150 and $100 \mathrm{eV}$ for the survey and the narrow scans, respectively. The step size was $1.12 \mathrm{eV}$ for the survey spectra and 0.078 for the narrow scans. Charge compensation was achieved with a flood gun of $4 \mathrm{eV}$ electrons. Data processing was achieved with a Winspec software, kindly supplied by the Laboratoire Interdisciplinaire de Spectroscopie d'Electrons (LISE, Namur, Belgium). The spectra were calibrated by using the N1s peak 
of PIBCA centred at $399.6 \mathrm{eV}$, a binding energy value reported by Beamson and Briggs [24] for poly (acrylonitrile) and poly (methacrylonitrile). The surface composition was determined using the manufacturer's sensitivity factors. The fractional concentration of a given element A (\% A) was computed by using equation 1 :

$$
\% A=\frac{\left(I_{A} / s_{A}\right)}{\sum\left(I_{n} / s_{n}\right)} \times 100 \%
$$

In which $I_{\mathrm{n}}$ and $S_{\mathrm{n}}$ are the integrated peak areas and the sensitivity factors, respectively.

\subsection{Size and zeta potential measurements:}

The size of the NPs was evaluated in MilliQ ${ }^{\circ}$ water by quasi-elastic light scattering using a Nanosizer N4 PLUS (Beckman-Coulter, Villepinte, France) operating at the angle of $90^{\circ}$. Zeta potential was measured in $1 \mathrm{mM} \mathrm{KCl}$ on a Zetasizer 4 (Malvern Instruments Ltd., Malvern, UK).

\subsection{Evaluation of complement activation by 2-D immunoelectrophoresis of C3:}

Protein C3 is a major plasma protein of the complement system circulating in functionally inactive form and involved in the non-specific recognition, i.e., opsonisation, of foreign bodies. A critical step of complement activation consists in the cleavage of C3 into a large fragment C3b and a small fragment C3a. Functionality of complement needs the presence of divalent ions. Thus, complement activation is assessed in human serum.

Its activation in the presence of different NPs was evidenced by evaluating conversion of C3 into C3b by 2-D immunoelectrophoresis using a polyclonal antibody to human $C 3[18,25]$. Human serum was obtained after calcifying plasma from healthy donors and stored at $-80^{\circ} \mathrm{C}$ until use. Veronal-buffered saline (VBS), VBS containing $0.15 \mathrm{mM} \mathrm{Ca}^{2+}$ and $0.5 \mathrm{mM} \mathrm{Mg}^{2+}$ ions $\left(\mathrm{VBS}^{2+}\right)$ and VBS containing $40 \mathrm{mM}$ ethylenediamine tetraacetic acid (VBS-EDTA) were prepared as described previously [26]. NPs $(200 \mu \mathrm{L})$ were incubated under gentle agitation for 1 hour at $37^{\circ} \mathrm{C}$ with $100 \mu \mathrm{L}$ human serum and $100 \mu \mathrm{L} \mathrm{VBS}{ }^{2+}$. To ensure a valid comparison of the different NPs, the $200 \mu \mathrm{L}$ sample volume of NPs contained an equal surface area of hydrated particles corresponding to $1,000 \mathrm{~cm}^{2}$. A NP relative surface area was calculated from the average hydrodynamic diameters according to [7].

After incubation, $5 \mu \mathrm{L}$ of each sample was subjected to a first electro-focusing electrophoresis on $1 \%$ agarose gel. The second-dimension electrophoresis was carried out on Gelbond ${ }^{\circledR}$ films in agarose gel plates containing a polyclonal antibody to human C3 (Complement C3 antiserum rose in goat, Sigma, France), recognizing both $\mathrm{C} 3$ and $\mathrm{C} 3 \mathrm{~b}$. The films were finally stained with Coomassie blue to reveal the presence of $\mathrm{C} 3$ and $\mathrm{C} 3 \mathrm{~b}$ which have reacted with the antibody (Sigma). Serum diluted in VBSEDTA $(1 / 4 \mathrm{~V} / \mathrm{v})$ was used as negative control of complement activation. Serum diluted in $\mathrm{VBS}^{2+}$ was used as control of the spontaneous activation of complement occurring in the experimental conditions used. Sephadex ${ }^{\circ}$ G 25 superfine (Pharmacia, Orsay, France) incubated in serum diluted in $\mathrm{VBS}^{2+}$ was used as a positive control. Each NP was analysed 3 times across the whole process. The height of the peaks showed on the immunoelectrophoretic plate corresponding to $\mathrm{C} 3$ and $\mathrm{C} 3 \mathrm{~b}$ respectively was measured 15 times. The results of the complement activation given by one type of NP were expressed as a percentage of $\mathrm{C} 3$ and $\mathrm{C} 3 \mathrm{~b}$ detected on the plate regarding the sum of the peaks height of $\mathrm{C} 3$ and $\mathrm{C} 3 \mathrm{~b}$. To highlight adsorption of C3 on NP surfaces, the sum of the peaks height of $\mathrm{C} 3$ and $\mathrm{C} 3 \mathrm{~b}$ was compared to the peak height of $\mathrm{C} 3$ given by the negative control (serum + VBS-EDTA) and expressed as a percentage of the height of this peak.

\subsection{Evaluation of proteins adsorption from citrated plasma by 2-D PAGE:}

Sample volumes with equivalent surface area $\left(3,000 \mathrm{~cm}^{2}\right)$ of NPs were incubated for 5 min at $37^{\circ} \mathrm{C}$ with $2.2 \mathrm{~mL}$ of citrate stabilised plasma (Rotes Kreuz, Berlin, Germany). NPs were separated from the 
medium by $23,000 \times \mathrm{g}$ for $1 \mathrm{~h}$ and washed three times with $1 \mathrm{~mL}$ bidistilled water. Proteins were desorbed from the NPs surface by $10 \mu \mathrm{L}$ of $10 \%$ sodium dodecyl sulphate (SDS) solution. The composition in desorbed proteins was analysed by 2-D PAGE [27]. The first electrophoresis performed in a $\mathrm{pH}$-gradient from 3 to 10 , consisted in a separation of proteins or protein fragments according to their isoelectric point by Immobiline DryStrips, pH 3-10 NL, $18 \mathrm{~cm}$ from Amersham Bioscience, whereas the second dimension of the electrophoresis led to separation of species according to their molecular weight within the range 6-250 kDa. Gels were then stained by silver staining protocol and scanned by laser densitometer. Gels were evaluated by software MELANIE II.

\section{$3 \quad$ RESULTS AND DISCUSSIONS}

\subsection{Chemical composition of the dry nanoparticles:}

Some NPs obtained by the redox radical polymerisation process, i.e. Hep-Rad and Dex71-Rad, and the polymers corresponding to the copolymer blocks constituting the particles, i.e. PIBCA, heparin, and dextran, were analysed by elemental analysis. PIBCA, Hep-Rad, Dex71-Hep-Rad and Dex71-Rad were also analysed by XPS.

In the survey scans of XPS, the main peaks were C1s, N1s and O1s, centred at ca 285, 400 and 531 $\mathrm{eV}$, respectively (data not shown). The spectral region between 0 and $270 \mathrm{eV}$ was flat in the case of PIBCA. Two small peaks at 168 and $233 \mathrm{eV}$, corresponding to S2p and S2s core levels, respectively, and the Na1s and NaKLL peaks of sodium, centred at 1072 and $496 \mathrm{eV}$, were present in the spectrum of Hep-Rad. These peaks were also present in the spectrum of Dex71-Hep-Rad. In the case of Dex71Rad, the 0-270 eV region was again flat. By comparison to PIBCA, all other spectra showed a significant decrease in the $\mathrm{C} 1 \mathrm{~s}$ relative intensity. This was also the case for the N1s peak. Conversely, the relative intensity of the O1s peak was increased.

The atomic composition of the different materials investigated was calculated from the results obtained either by elemental analysis (EA), or by XPS (Table 1). They were expressed in Table 2 as the atomic ratios $\mathrm{O} / \mathrm{C}$ and $\mathrm{N} / \mathrm{C}$, and compared with those calculated from the theoretical formulas of PIBCA, dextran and heparin. The atomic composition of whole polymers is provided by EA, whereas contribution of atoms present at the NP surface should be increased in the composition given by XPS. Comparison between the results obtained by these two methods should provide evidence about a preferential distribution of the polysaccharide on the NP surface. Indeed, the O/C atomic ratio would be dramatically affected by the presence of dextran and heparin since it could increase from a theoretical value of 0.25 for pure PIBCA to 0.83 and 1.58 for pure dextran and heparin, respectively. Similarly, the N/C atomic ratio was expected to decrease for PIBCA NPs coated with polysaccharides.

Table 1: Composition of uncoated and coated PIBCA samples (in atomic \%)

\begin{tabular}{|l|c|c|c|c|c|c|c|c|c|c|}
\hline & \multicolumn{2}{|c|}{ C } & \multicolumn{2}{c|}{ N } & \multicolumn{2}{c|}{ o } & \multicolumn{2}{c|}{ S } & \multicolumn{2}{c|}{ Na } \\
\hline Sample & EA & XPS & EA & XPS & EA & XPS & EA & XPS & EA & XPS \\
\hline PIBCA & $72.7 \pm 0.6$ & 71.7 & $9.0 \pm 0.1$ & 8.52 & $18 \pm 1$ & 19.8 & nd & 0 & nd & 0 \\
\hline Heparin & $30.0 \pm 0.5$ & $\mathrm{nd}$ & $2.41 \pm 0.05$ & $\mathrm{nd}$ & $55 \pm 4$ & $\mathrm{nd}$ & $4.20 \pm 0.05$ & $\mathrm{nd}$ & $8.1 \pm 0.5$ & $\mathrm{nd}$ \\
\hline Hep-Rad & $64 \pm 2$ & 64.0 & $7.7 \pm 0.4$ & 7.14 & $25.40 \pm 0.01$ & 25.4 & $0.4 \pm 0.2$ & 1.02 & $2.7 \pm 0.5$ & 2.43 \\
\hline Dextran & $50.0 \pm 0.5$ & $\mathrm{nd}$ & $\mathrm{nd}$ & $\mathrm{nd}$ & $50 \pm 1$ & $\mathrm{nd}$ & $\mathrm{nd}$ & $\mathrm{nd}$ & $\mathrm{nd}$ & $\mathrm{nd}$ \\
\hline Dex71-Hep-Rad & $\mathrm{nd}$ & 62.5 & $\mathrm{nd}$ & 5.78 & $\mathrm{nd}$ & 30.4 & $\mathrm{nd}$ & 0.98 & $\mathrm{nd}$ & 0.31 \\
\hline Dex71-Rad & $67.9 \pm 0.9$ & 65.7 & $7.29 \pm 0.04$ & 5.8 & $24.80 \pm 0.04$ & 28.5 & $\mathrm{nd}$ & 0 & $\mathrm{nd}$ & 0 \\
\hline
\end{tabular}

nd: not determined 
Table 2: Composition in atomic ratios $\mathrm{O} / \mathrm{C}$ and $\mathrm{N} / \mathrm{C}$ calculated from the theoretical formulas of PIBCA $\left(\mathrm{C}_{8} \mathrm{NO}_{2}\right)_{n}$, dextran $\left(\mathrm{C}_{6} \mathrm{O}_{5}\right)_{n}$ and heparin $\left(\mathrm{C}_{12} \mathrm{NO}_{19} \mathrm{~S}_{3} \mathrm{Na}_{4}\right)_{n}$ with those determined by EA and XPS in the different samples. Since hydrogen is not detected in XPS, hydrogen was not taken into account in the theoretical formulas.

\begin{tabular}{|l|l|l|l|l|l|l|}
\hline Sample & \multicolumn{5}{l}{ O/C } & \multicolumn{2}{l|}{ N/C } \\
\hline & Theor & EA & XPS & Theor & EA & XPS \\
\hline PIBCA & 0.25 & 0,248 & 0,276 & 0.125 & 0,124 & 0,119 \\
\hline Heparin & 1.58 & 1,833 & - & 0.083 & 0,080 & - \\
\hline Hep-Rad & - & 0,397 & 0,397 & - & 0,120 & 0,112 \\
\hline Dextran & 0.833 & 1,000 & - & 0,000 & 0,000 & - \\
\hline Dex71-Hep-Rad & - & - & 0,486 & - & - & 0,092 \\
\hline Dex71-Rad & - & 0,365 & 0,434 & - & 0,107 & 0,088 \\
\hline
\end{tabular}

Results obtained from the analysis of the homopolymers were generally in good agreement with the theoretical values calculated from the chemical formulae. However, the analysis of the NPs showed that a significant difference between the O/C ratios determined by EA and XPS clearly appeared only in the case of Dex71-Rad. The N/C was found to decrease. This decrease was even observed for particles containing heparin which includes nitrogen atoms. In the case of these NPs the detection of sulphur and sodium confirmed the presence of heparin on the surface. Taken together, the results of XPS measurements indicated that dextran and/or heparin were located at the surface of the particles. The rather small differences between the results from EA and XPS could be explained by the fact that the NPs made of polymers were dry and small in size, especially the Hep-Rad ones. It can be hypothesised that the thickness of the layer analysed by XPS took into account a significant contribution of the NP core. According to this hypothesis, the limits of the XPS analysis as applied for NP surface chemical composition determinations were reached. This can also be explained by the fact that NPs, which are already small in diameter with the hydrated shell as measured by quasielastic light scattering, may show a shell totally collapsed on the NP core after drying further reducing the thickness of the shell contribution analysed by XPS. In another way, the XPS results could also suggest that the NP structure differed from a typical core-shell structure as previously hypothesised and could result from a multilayer arrangement of the copolymers within the NPs to give an onion-like structure.

\subsection{Physicochemical characteristics of the particles:}

The size and zeta potential of the NPs prepared with the different polysaccharides are given in Table 3. Comparison of the hydrodynamic diameters measured for the NPs prepared with either dextran 15 $\mathrm{kDa}$ or dextran $71 \mathrm{kDa}$, and by either redox radical or anionic polymerisation, showed that the NPs diameter was affected both by the molecular weight of the polysaccharide and by the type of polymerisation process. Dex15-Rad particles were smaller than the Dex71-Rad ones. This fact was likely related to the two different lengths of the dextran chains which composed the shell of the particles. NPs prepared by anionic polymerisation in the presence of dextran $71 \mathrm{kDa}$ were twice smaller than those prepared by radical polymerisation in the presence of the same amount of the same dextran. This fact was related to the likely different outermost structures of the NP surface. Indeed, depending on the initiation of the polymerization of IBCA, it can be expected that the structure of the NP shell consisted on polysaccharide chains arranged as "loops" and "trains" or as a "brush" as suggested in previous works [21, 28]. The particles prepared by anionic polymerisation in the presence of dextran $15 \mathrm{kDa}$ were not submicronic and tended to aggregate, in opposition to Dex15-Rad NPs. This can be explained by a difference of colloid stabilization efficiency of the copolymer depending on the mechanism of polymerization used to synthesize the NPs. This hypothesis is supported by the fact that copolymers of the same composition in IBCA and 
polysaccharide synthesized either by redox radical polymerization or by the anionic polymerization were shown to display very different capacity to stabilize an emulsion [29].

Table 3: Hydrodynamic diameter and zeta potential of the PIBCA nanoparticles prepared with the different polysaccharides

\begin{tabular}{|l|l|l|l|l|}
\hline \multirow{2}{*}{ Samples } & \multicolumn{2}{|l|}{ Size } & $\begin{array}{l}\text { Zeta Potential } \\
(\mathrm{mV})\end{array}$ \\
\cline { 2 - 5 } & $\begin{array}{l}\mathrm{D}_{\mathrm{H}} \\
(\mathrm{nm})\end{array}$ & $\begin{array}{l}\text { Width } \\
(\mathrm{nm})\end{array}$ & $\begin{array}{l}\text { Polydispersity } \\
\text { Index }\end{array}$ & \\
\hline Dex71-Rad & $297 \pm 5$ & $80 \pm 8$ & 0.105 & $-11 \pm 2$ \\
\hline Dex15-Rad & $200 \pm 4$ & $47 \pm 5$ & 0.074 & $-19 \pm 3$ \\
\hline Hep-Rad & $93 \pm 3$ & $33 \pm 2$ & 0.259 & $-48 \pm 3$ \\
\hline Dex71-Hep-Rad & $186 \pm 4$ & $67 \pm 7$ & 0.267 & $-24 \pm 2$ \\
\hline Dex-Sulph-Rad & $274 \pm 3$ & $64 \pm 6$ & 0.072 & $-42 \pm 3$ \\
\hline Dex71-An & $152 \pm 3$ & $54 \pm 6$ & 0.253 & $-9 \pm 2$ \\
\hline Dex15-An & $\geq 2,000$ & nd & nd & nd \\
\hline
\end{tabular}

$D_{H}$ (Mean hydrodynamic diameter) and Zeta potential: Average value from three determinations performed on different batches of nanoparticles, Width: width of the size distribution, nd: not determined

NPs prepared from heparin were the smallest ones and those prepared from a mixture of dextran 71 $\mathrm{kDa}$ and heparin showed an intermediate diameter between NPs prepared from the corresponding single polysaccharides. Interestingly, there was only one population concerning these NPs prepared from a blend of polysaccharides. Dex-Sulph-Rad NPs showed a diameter close to the one of Dex71Rad NPs.

The zeta potential of the NPs prepared with dextran, which is a neutral polysaccharide, showed a slightly negative value which was affected mainly by the molecular weight of the dextran used. However, in the case of the NPs prepared in the presence of dextran $71 \mathrm{kDa}$, these obtained by anionic polymerisation were endowed with a zeta potential closer to neutrality than those obtained by radical polymerisation. Here again, the difference observed between NPs obtained by the two different polymerizations can be explained by a difference in the arrangement of the polysaccharide chains in the shell of the NPs. It seems that the arrangement in "loops" and "trains" expected in the case of the anionic polymerization formed better mask for a contribution of the core to zeta potential than the arrangement in "brush" expected in the case of the redox radical polymerization.

As expected, the NPs prepared either from heparin or from dextran sulphate, which are negatively charged polysaccharides, displayed a very negative zeta potential value. An intermediate value of zeta potential was obtained for the NPs prepared from a blend of dextran $71 \mathrm{kDa}$ and heparin, when compared with the NPs prepared with the corresponding single polysaccharides. Taken together the data concerning size and zeta potential of the NPs prepared from a blend of polysaccharides, suggested that the suspension contained a single population of NPs coated with both polysaccharides.

\subsection{Activation of $\mathrm{C} 3$ in the presence of the nanoparticles:}

The results of the 2D immunoelectrophoresis of $\mathrm{C} 3$ and $\mathrm{C} 3 \mathrm{~b}$ performed to evaluate the degree of complement activation induced by the NPs after incubation in serum containing calcium and magnesium ions are given in Table 4 and illustrated in Figure 1.

Concerning the dextran-coated particles, the level of complement activation ranged from very low to high. Particles prepared from dextran $15 \mathrm{kDa}$ were strong activators whatever the type of polymerisation process, evidencing a lack of protection against complement activation by the short dextran chains, either "in brush or "in loops" and "trains" conformations. 
Table 4: C3 activation and adsorption after 60 min incubation of $1,000 \mathrm{~cm}^{2}$ of samples with $100 \mu \mathrm{L}$ of human serum at $37^{\circ} \mathrm{C}$ (total volume after dilution $400 \mu \mathrm{L}$ ), as measured by 2-D immunoelectrophoresis and expressed as the relative heights of the $\mathrm{C} 3$ and $\mathrm{C} 3 \mathrm{~b}$ peaks.

\begin{tabular}{|l|l|l|l|l|}
\hline Sample & $\begin{array}{l}\text { C3 } \\
(\%)\end{array}$ & $\begin{array}{l}\text { C3b } \\
(\%)\end{array}$ & $\begin{array}{l}\text { C3 +C3b } \\
\text { recovered* (\%) }\end{array}$ & Comments \\
\hline Serum-VBS-EDTA & 100 & 0 & 100 & $\begin{array}{l}\text { Absence of divalent ions, } \\
\text { negative control }\end{array}$ \\
\hline Serum-VBS ${ }^{2+}$ & 83 & 17 & 98 & Spontaneous activation \\
\hline Sephadex & $64 \pm 5$ & $36 \pm 5$ & 98 & Reference activator \\
\hline Dex71-Rad & $80 \pm 2$ & $20 \pm 2$ & 100 & $\begin{array}{l}\text { Similar to spontaneous } \\
\text { activation }\end{array}$ \\
\hline Dex15-Rad & $16 \pm 1$ & $84 \pm 1$ & 112 & Strong activation \\
\hline Hep-Rad & $100 \pm 2$ & 0 & 36 & No activation + Adsorption \\
\hline Dex71-Hep-Rad & $100 \pm 3$ & 0 & 57 & No activation + Adsorption \\
\hline Dex-Sulph-Rad & 0 & 0 & 0 & High adsorption \\
\hline Dex71-An & $5 \pm 5$ & $95 \pm 5$ & 106 & Strong activation \\
\hline Dex15-An & 0 & $100 \pm 5$ & 91 & Strong activation \\
\hline
\end{tabular}

Hundred percent corresponds to the sum of the heights of both peaks (Mean of 15 determinations).

$* \mathrm{C} 3+\mathrm{C} 3 \mathrm{~b}$ recovered relative to peak height observed in the negative control: serum-VBS-EDTA

NPs prepared from dextran $71 \mathrm{kDa}$ by radical polymerisation were very low activators, whereas NPs prepared from the same dextran by anionic polymerisation were strong activators. Concerning complement activation, the outermost structure of Dex71-An NPs behaved like crosslinked dextran, i.e., Sephadex ${ }^{\star}$, known as a strong activator [17], whereas the outermost structure of Dex71-Rad behaved like soluble dextran. As the activation of complement is an amplified process, small variations in the surface structure of NPs can result in striking differences in complement activating capacities, as already demonstrated with PLA-PEO NPs covered with different amounts of PEO [7], or with PIBCA NPs covered with PEO in different conformations [13].

A single peak corresponding to non-activated C3 was detected in the case of the Hep-Rad NPs. This result agreed with the complement activation inhibition properties of heparin [30]. It also agreed with the results reported by Passirani et al. [18] considering heparin coated PMMA NPs. However, the height of the C3 peak was decreased and because these plates did not show any C3b, the total amount of complement recovered was reduced suggesting that some of $\mathrm{C} 3$ remained adsorbed on the NP surface. This was confirmed considering the percentage of C3 recovered from these NPs. It is also supported by the presence of a shoulder on the left side hand of the $\mathrm{C} 3$ peak appearing just over the well indicated by the cross-section of the arrows on the 2D immunoelectrophoretic plates (Figure 1). To further investigate this hypothesis, the complement activation induced by NPs coated with dextran sulphate was studied. Indeed, C3 was found to adsorb strongly on surfaces bearing sulphonate groups such as polyacrylonitrile haemodialysis membranes [31] and a poly (2-acrylamido 2-methylpropane sulphonate)-grafted polystyrene surface [32]. Thus, dextran sulphate was used to produce NPs on which a model polysaccharide having high potential complement adsorption capacity would form the shell. On these model NPs, no peaks of either C3 or C3b could be detected by $2 \mathrm{D}$ immunoelectrophoresis. This confirmed that a strong adsorption of the complement proteins at the NP surface led to a reduction of the peak height of those components on the immunoelectrophoretic plate. NPs coated with a blend of dextran $71 \mathrm{kDa}$ and heparin, were also shown to avoid complement activation. Adsorption of C3 was lower, probably because surface density of heparin was lower than in NPs prepared from heparin alone. 

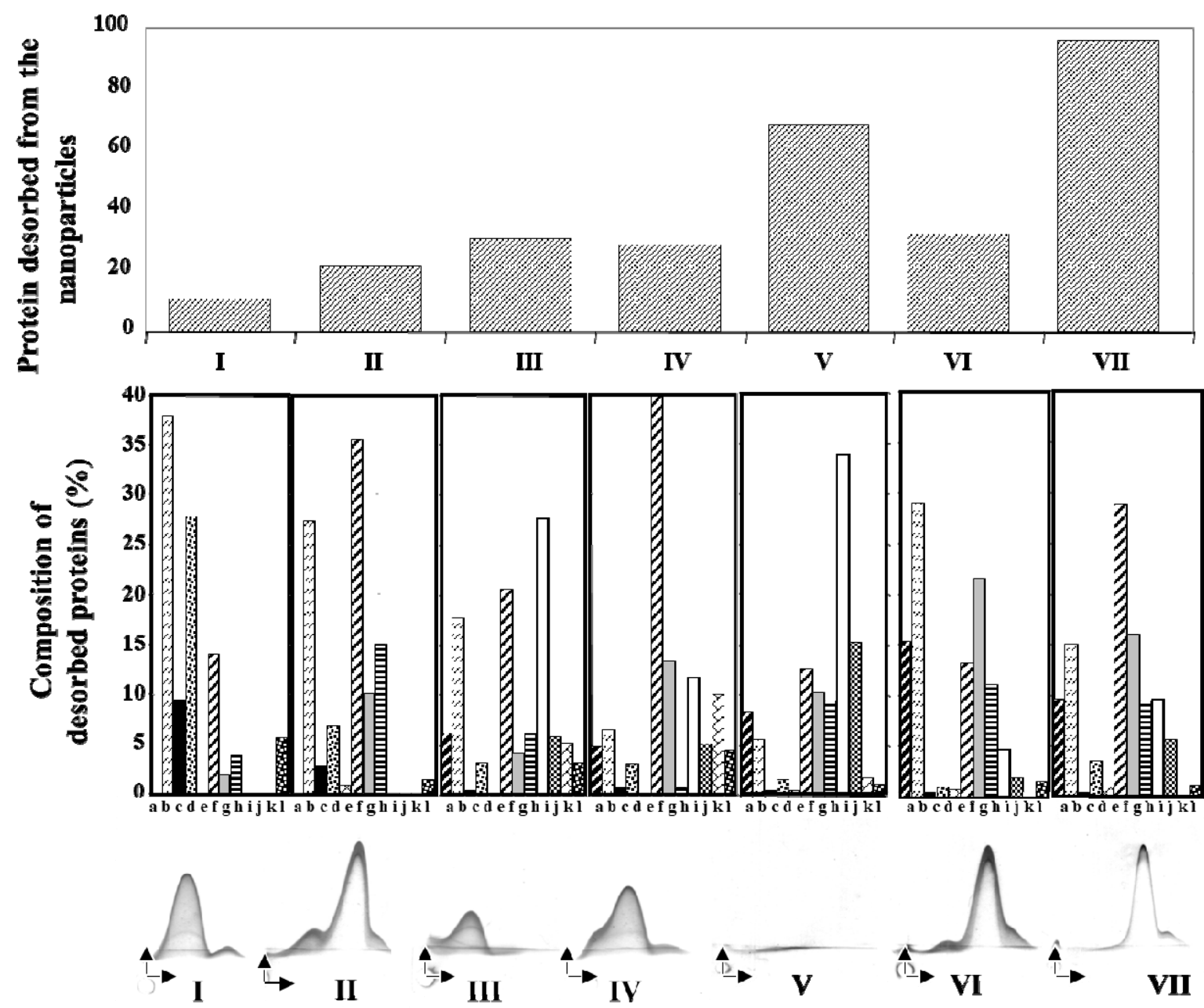

Figure 1: Summary of the interactions between the core-shell NPs and citrated blood plasma proteins (upper and middle lanes) and NPs and complement (lower lane). Sample volumes with equivalent surface area $\left(3,000 \mathrm{~cm}^{2}\right)$ of NPs were incubated for $5 \mathrm{~min}$ at $37^{\circ} \mathrm{C}$ with $2.2 \mathrm{~mL}$ of human citrated plasma. After centrifugation, proteins were desorbed from the particles surface by $10 \%$ SDS-solution. Composition of the desorbed mixture was analysed by 2-D PAGE. The results displaying the total amount of desorbed proteins or protein fragments are presented in the upper lane (arbitrary unit). Composition of each mixture is given in the middle lane (percentage related to the results of the upper lane). Sample volumes with equivalent surface area $\left(1,000 \mathrm{~cm}^{2}\right)$ of NPs were incubated for 60 min at $37^{\circ} \mathrm{C}$ with human serum diluted $1: 4$ in $V B S^{2+}$. Then each sample was subjected to 2-D immunoelectrophoresis of C3 antigens. Start of migration is indicated by arrows. The relative amounts of $\mathrm{C} 3$ and $\mathrm{C} 3 \mathrm{~b}$ are indicated by the first and second peak respectively. NPs samples are Dex71-Rad (I), Dex15-Rad (II), Hep-Rad (III), Dex71-Hep-Rad (IV), Dex-Sulph-Rad (V), Dex71-An (VI) and Dex15-An (VII). Proteins and protein fragments are Albumin (a), ApoA-I (b), ApoA-2 (c), ApoC-2 (d), ApoJ (e), Fibrinogen $\alpha$ chain (f), Fibrinogen $\beta$ chain (g), Fibrinogen $\gamma$ chain (h), IgG $\gamma$ chain (i), Ig light chain (j), Serotransferrine ( $k$ ) and Transthyretine (I).

\subsection{Proteins adsorption from citrated plasma:}

A detailed analysis of the serum proteins which adsorbed on the NPs surface after incubation in citrated serum was performed by 2D PAGE after recovery of the proteins and protein fragments from the NPs surface using a surfactant, i.e., SDS [27]. In these experiments, citrated plasma was used to investigate simple adsorption phenomena and to avoid the occurrence of amplified phenomena 
requiring the presence of divalent ions such as complement activation and coagulation. The results showing the types and the amounts of proteins or protein fragments desorbed from the NPs are presented in Table 5 and in Figure 1.

Table 5: Proteins and protein fragments desorbed by SDS from equivalent surface areas of nanoparticles, after 5 min contact with citrated plasma at $37^{\circ} \mathrm{C}$.

\begin{tabular}{|c|c|c|c|c|c|c|c|c|c|}
\hline \multirow{3}{*}{ Sample } & \multirow{3}{*}{ 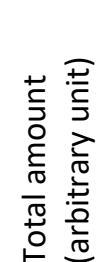 } & \multicolumn{8}{|c|}{ Predominant protein or fragment } \\
\hline & & \multicolumn{2}{|l|}{ First } & \multicolumn{2}{|l|}{ Second } & \multicolumn{2}{|l|}{ Third } & \multicolumn{2}{|l|}{ Fourth } \\
\hline & & Name & 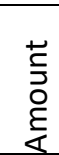 & Name & 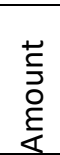 & Name & 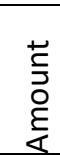 & Name & 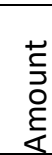 \\
\hline Dex71-Rad & 11 & ApoA-I & 4 & ApoC-II & 3 & Fg- $\alpha$-chain & 2 & & \\
\hline Dex15-Rad & 22 & Fg- $\alpha$-chain & 8 & ApoA-I & 6 & $\mathrm{Fg}-\gamma$-chain & 3 & Fg- $\beta$-chain & 2 \\
\hline Hep-Rad & 32 & $\lg G$ & 9 & Fg- $\alpha$-chain & 6 & ApoA-I & 6 & $\begin{array}{l}\text { Albumin } \\
\text { Fg- } \gamma \text {-chain }\end{array}$ & 2 \\
\hline $\begin{array}{l}\text { Dex71-Hep- } \\
\text { Rad }\end{array}$ & 30 & Fg- $\alpha$-chain & 12 & Fg- $\beta$-chain & 4 & IgG- $\gamma$-chain & 3 & Serotransferrin & 3 \\
\hline $\begin{array}{l}\text { Dex-Sulph- } \\
\text { Rad }\end{array}$ & 68 & IgG- $\gamma$-chain & 23 & Ig light chain & 10 & Fg- $\alpha$-chain & 9 & Fg- $\beta$-chain & 7 \\
\hline Dex71-An & 33 & ApoA-I & 9 & Fg- $\beta$-chain & 7 & Albumin & 5 & Fg- $\alpha$-chain & 4 \\
\hline Dex15-An & 96 & Fg- $\alpha$-chain & 28 & Fg- $\beta$-chain & 15 & ApoA-I & 14 & Albumin & 9 \\
\hline
\end{tabular}

The smallest total amount of desorbed proteins was obtained for the NPs prepared from dextran 71 kDa by radical polymerisation, i.e., Dex71-Rad. The amount desorbed from NPs prepared from the same dextran but by anionic polymerisation was about three times higher. Even if the difference in protein adsorption was not as striking as the difference in complement activation, this result demonstrated that the outermost surface of Dex71-Rad was more protective against protein adsorption than the surface of Dex71-An. The amounts of proteins desorbed from Dex15-Rad NPs was twice more than the amount desorbed from Dex71-Rad, whereas the amount desorbed from Dex15-An was over four times more than Dex15-Rad. It can be concluded that the protective effect against protein adsorption by steric repulsion resulting from the dextran chains of different molecular weight, either "in brush", or "in loops" and "trains", was the most efficient in the case of the longest dextran chain "in brush" (Dex71-Rad), whereas it was quite inefficient in the case of the shortest dextran in "loops" and "trains" (Dex15-An).

Concerning the particles prepared from heparin alone and from a blend of heparin and dextran 71 $\mathrm{kDa}$, the amounts desorbed were similar and about three times higher than the amount desorbed from Dex71-Rad and about one and a half times higher than the amount desorbed from Dex15-Rad. As the average length of heparin could be compared to the length of dextran $15 \mathrm{kDa}$, the repulsive effect of both types of "brushes" could be compared. However, heparin is known to be endowed with affinity for many proteins, resulting in higher adsorption. In the case of Dex71-Hep-Rad NPs, the steric repulsive effect resulting from the rather short "brush" of heparin was not improved by the presence of the longest dextran chains, probably due to the high affinity of proteins for heparin. The amounts of proteins desorbed from particles prepared from dextran sulphate was six times more than the amount desorbed from Dex71-Rad NPs and about twice the amount desorbed from HepRad or Dex71-Hep-Rad NPs. This was observed despite the fact that the chains of dextran sulphate were about twice longer than the heparin chains and could be more efficient concerning the contribution to the steric repulsion of proteins from the NP surface. This result obtained in citrated plasma confirmed the strong adsorption behaviour of the NPs coated with dextran sulphate already observed during the $\mathrm{C} 3$ experiments perfomed in serum containing divalent ions. 
It was attempted to correlate the results of desorption of individual proteins or protein fragments from each NP sample to the nature of the polysaccharide forming the shell of the NP and to the method of polymerization. Concerning the dextran-coated NPs, the first predominant protein found after desorption from Dex71-Rad and Dex71-An was ApoA-I. This protein represented about one third of the total amount of proteins desorbed from these NPs, whatever the mechanism of polymerisation. ApoA-I was also found on Dex15-Rad as second protein and on Dex15-An and HepRad as third protein, but not on the other NPs. ApoA-I has been identified as a major adsorbate on biomaterial surfaces after plasma contact, but its importance concerning biocompatibility is still unknown [33].

The $\alpha$ chain of fibrinogen was the first predominant protein fragment desorbed from Dex15-Rad and Dex15-An, but its presence on Dex71-An was found as fourth protein and was hardly detected on Dex71-Rad. Adsorption of fibrinogen and ApoA-I on the dextran-coated particles is likely not specific. Fibrinogen is a larger protein than ApoA-I. Providing that the steric repulsive effect would be more efficient towards large proteins, only small proteins could adsorb on the surface of the wellprotected Dex71-coated NPs, whereas fibrinogen could adsorb on the less protected Dex15-coated NPs. This hypothesis could be supported by the fact that the total adsorption is lower on Dex-Rad NPs than on Dex-An particles which are less protected.

Concerning the negatively charged NPs, IgG and some fragments were found in large amount on DexSulph-Rad, twice less on Hep-Rad and in low amount on Dex71-Hep-Rad. The $\alpha$ chain of fibrinogen was also found as first predominant protein on Dex71-Hep-Rad and in smaller amounts on DexSulph-Rad and Hep-Rad. ApoA-I was detected on Hep-Rad. No obvious correlation was highlighted between the adsorption patterns of the plasma proteins and the physicochemical properties of the nanoparticles. The adsorption of proteins on such surfaces resulted from a combination of several phenomena including steric repulsion, ionic interactions and affinity between proteins and polysaccharides. Thus, the results obtained in this study suggested that each type of NPs showed a unique protein adsorption pattern.

\section{CONCLUSIONS}

In this paper, the in vitro interactions of polysaccharide-coated NPs with blood proteins were investigated either in serum, or in citrated plasma. The type and the length of the polysaccharides used to prepare the NPs differed as well as the mechanisms of the initiation of the polymerisation of IBCA resulting in NPs with different surface properties (charge, structure).

XPS used to characterize the composition of the NP surface confirmed the presence of polysaccharide. Zeta potential varied greatly according to the nature and composition of the polysaccharides. With neutral polysaccharides, zeta potential also depended on the method of polymerization and in the case of the redox radical polymerization it varied with the molecular weight of the polysaccharides. This was attributed to a difference in the arrangement of the polysaccharide chains at the NP surface.

Differences between NPs were more clearly evidenced when considering their interactions with blood proteins. An effect of both the method of polymerization and of the characteristics of the polysaccharides on the NPs surface was evidenced considering complement activation induced by the NPs. It can lead to totally opposite results considering a single modification of molecular weight or of the method of polymerization. In citrated plasma, the interactions resulted from a combination of steric repulsion and affinity. Efficacy of steric repulsion was noted with dextran coated NPs decreasing in the order: Dex71-Rad > Dex15-Rad > Dex71-An >> Dex15-An. Conversely, the effect of the affinity was revealed with NP coated with sulphonate containing polysaccharides (heparin, dextran sulphate). However, no clear correlation could be drawn considering the composition of the proteins adsorbed in relation with the nature and the molecular weight of the polysaccharide at the NP surface as well as with the method of NP preparation. Indeed, each type of NPs showed a unique 
protein adsorption pattern suggesting an exquisite sensitivity of blood proteins to rather small differences in both the structure and the characteristics of the polysaccharides grafted on the NPs surface. As a consequence, this could result in dramatic effects on their interactions with blood proteins, especially in conditions in which the effects are amplified as in the case of complement activation. This may also dramatically affect the in vivo fate of the NPs after intravenous administration. Much work is still required to better understand how the physico-chemical characteristics of the NPs surface are influencing the interactions with blood proteins. Also, new techniques of characterization of the NPs surface are needed to better investigate the properties of the hydrophilic chains located at the NPs surface.

\section{ACKNOWLEDGEMENTS}

The authors want to thank Dr. K. Broadley from Loctite for his kindness in providing isobutyl cyanoacrylate monomer and Mr. Pascal Bargiela (University Paris VII) for assistance with XPS measurements. Cédric Chauvierre was a fellow of the Ministry of Research in France.

\section{REFERENCES}

[1] Archambault JC, Brash JL. Protein resistant polyurethane surfaces by chemical grafting of PEO: aminoterminated PEO as grafting reagent. Coll. Surf. B Biointerfaces 2004; 25:9-16.

[2] Kwon GS Diblock copolymer nanoparticles for drug delivery. Critical Reviews in Therapeutic Drug Carrier Systems 1998; 15: 481-512.

[3] Vauthier C, Fattal E, Labarre D. From polymer chemistry and physicochemistry to nanoparticulate drug carrier design and applications. In: Yaszemski MJ, Trantolo DJ, Lewandrowski KU, Hasirci V, Altobelli DE, Wise DL, editors. Tissue engineering and novel delivery systems. M. Dekker, Inc., New York, Basel, 2004, p 563-98.

[4] Gref R, Minamitake Y, Peracchia MT, Trubetskoy V, Torchilin V, Langer R. Biodegradable long-circulating polymeric nanospheres. Science 1994; 263: 1600-3.

[5] Bazile D, Prud'homme C, Bassoullet MT, Marlard M, Spenlehauer G, Veillard M. Stealth Me.PEG-PLA nanoparticles avoid uptake by the mononuclear phagocytes system. J Pharm Sci 1995; 84: 493-8.

[6] Gref R, Lück M, Quellec P, Marchand M, Dellacherie E, Harnish S, Blunk T, Müller RH. "Stealth" core-corona nanoparticles surface modified by poly ethylene glycol (PEG): influence of the corona (PEG chain length and surface density) and of the core composition on phagocytic uptake and plasma protein adsorption. Colloids and Surfaces B: Biointerfaces 2000; 18: 301-13.

[7] Vittaz M, Bazile D, Spenlehauer G, Verrechia T, Veillard M, Puisieux F, Labarre D. Effect of PEO surface density on long-circulating PLA-PEO nanoparticles which are very low complement activators. Biomaterials 1996; 17: 1575-81.

[8] Sahli H, Tapon-Bretaudiere J, Fischer AM, Sternberg C, Spenlehauer G, Verrecchia T, Labarre D. Interactions of poly (lactic acid) and poly (lactic acid-co-ethylene oxide) nanoparticles with the plasma factors of the coagulation system. Biomaterials 1997; 18: 281-[9] Torchilin VP, Trubetskoy VS. Which polymers can make nanoparticulate drug carriers long-circulating? Adv. Drug Delivery Rev 1995; 16: 141-155.

[10] Weissleder R, Bogdanov A, Neuwelt EA, Papisov M. Long-circulatinf iron oxides for MR imaging. Adv. Drug Delivery Rev 1995; 16: 321-334.

[11] Devine DV, Bradley AJ. The complement system in liposome clearance: can complement deposition be inhibited? Adv. Drug Delivery Rev 1998; 32: 19-29.

[12] Österberg E, Bergström K, Holmberg K, Schuman TP, Riggs JA, Burns NL, Van Alstine JM, Harris JM. Proteinrejecting ability of surface-bound dextran in end-on and side-on configurations: comparison to PEG. J Biomed Mater Res 1995; 29: 741-7.

[13] Peracchia MT, Vauthier C, Passirani C, Couvreur P, Labarre D. Complement consumption by poly (ethylene glycol) in different conformations chemically coupled to poly (isobutyl 2-cyanoacrylate) nanoparticles. Life Sci 1997; 61: 749-61.

[14] Blondin C, Bataille I, Letourneur D. Polysaccharides for vascular cell targeting. Critical Reviews in Therapeutic Drug Carrier Systems 2000; 17: 327-75.

[15] Sihorkar V, Vyas SP. Potential of polysaccharide anchored liposomes in drug delivery, targeting and immunization. J Pharm Pharmaceut Sci 2001; 4: 138-58. 
[16] Passirani C, Barratt G, Devissaguet JP, Labarre D. Long-circulating nanoparticles bearing heparin or dextran covalently bound to poly (Methyl Methacrylate). Pharm Res 1998; 15: 1046-50.

[17] Carreno MP, Labarre D, Jozefowicz M, Kazatchkine MD. The ability of Sephadex to activate human complement is suppressed in specifically substituted functional Sephadex derivatives. Mol Immunol 1988; 25: 165-71.

[18] Passirani C, Barratt G, Devissaguet JP, Labarre D. Interactions of nanoparticles bearing heparin or dextran covalently bound to poly (Methyl Methacrylate) with the complement system. Life Sci 1998; 62: 775-85.

[19] Lemarchand C. Développement de nanoparticules coeur (polyester) - couronne (polysaccharide).Ph. D. Thesis, University Paris XI-South, 18 May 2004

[20] Lemarchand C., Couvreur P, Besnard M, Costantini D, Gref R. Novel polyester-polysaccharide nanoparticles. Pharm. Res. $2003 ; 20$ : 1284-92.

[21] Chauvierre C, Labarre D, Couvreur P, Vauthier C. Radical emulsion polymerization of alkylcyanoacrylates initiated by the redox system dextran-cerium(IV) under acidic aqueous conditions. Macromolecules 2003; 36: 6018-27.

[22] Chauvierre C, Labarre D, Couvreur P, Vauthier C. Novel polysaccharide-decorated poly (alkylcyanoacrylate) nanoparticles. Pharm Res 2003; 20: 1786-93.

[23] Couvreur P, Kante B, Roland M, Guiot P, Bauduin P, Speiser P. Polycyanoacrylate nanocapsules as potential lysosomotropic carrier: preparation, morphological and sorptive properties. J Pharm Pharmacol 1979; 31: 331-2.

[24] Beamson G, Briggs D. editors. High Resolution XPS of Organic Polymers. The Scienta ESCA300 Database, John Wiley, Chichester 1992.

[25] Laurell CB. Quantitative estimation of proteins by electrophoresis in agarose gels containing antibodies. Anal Biochem 1966; 15: 45-52.

[26] Kazatchkine MD, Hauptmann G, Nydegger UE. Techniques du complément, INSERM, Paris, 1986, pp 22-23

[27] Blunk T, Hochstrasser DF, Sanchez JC, Muller BW, Muller RH. Colloidal carriers for intravenous drug targeting: plasma protein adsorption patterns on surface-modified latex particles evaluated by twodimensional polyacrylamide gel electrophoresis. Electrophoresis 1993; 14: 1382-7.

[28] Chauvierre C, Vauthier C, Labarre D, Hommel H. Evaluation of the surface properties of dextran-coated poly(isobutylcyanoacrylate) nanoparticles by spin-labelling coupled with electro resonance spectroscopy. Colloid Polym Sci 2004; 282: 1016-25.

[29] Chauvierre C, Labarre D, Couvreur P, Vauthier C. A new approach for the characterization of insoluble amphiphilic copolymers based on their emulsifying properties Colloid Polym Sci 2004; 282: 1097-1104.

[30] Kazatchkine MD, Fearon D, Silbert J, Austen K. Surface-associated heparin inhibits zymosan-induced activation of the human alternative pathway by augmenting the regulatory action of the control proteins on particle-bound C3b. J Exp Med 1979; 150: 1202-15

[31] Janatova J, Cheung AK, Parker CJ. Biomedical polymers differ in their capacity to activate complement. Complement Inflamm 1991; 8: 61-9.

[32] Murakami Y, Iwata H, Kitano E, Kitamura H, Ikada Y. Interaction of poly (2-acrylamido 2-methylpropane sulfonate)-grafted polystyrene beads with cationic complement proteins. J Biomater Sci Polym Ed 2001; 12: 451-65.

[33] Cornelius RM, Archambault J, Brash JL. Identification of apolipoprotein A-I as a major adsorbate on biomaterial surfaces after blood or plasma contact. Biomaterials 2002; 23: 3583-7. 